\title{
Chronic Allograft Nephropathy (Chronic Allograft Damage): Can It Be Avoided?
}

\author{
Serdar Yilmaz
}

Published online: 2 April 2014

(C) Springer International Publishing AG 2014

\begin{abstract}
Although there has been significant progress over the last three decades in renal transplantation, which has been driven primarily by improvements in short-term graft survival, there has been little parallel improvement in long-term outcomes. Advances in histological indicators of chronic allograft pathology have the potential to allow for earlier intervention as well as to provide surrogate endpoints for drug development. Identification of risk factors such as the presence of acute rejection episodes, ischemia/reperfusion injury, donor and recipient age considerations, hypertension, hyperlipidemia, glomerular hyperfiltration, and cytomegalovirus infection will aid in the development of strategies and treatments to mitigate these risks. Of particular importance is the optimization of immunosuppressive regimens to prevent acute rejection episodes. Despite our deeper understanding of clinical risk factors, it is clear that other determinants of graft loss have yet to be identified and that other "missing" factors must play a role in the pathogenesis of late kidney graft loss, highlighting the need for effective therapies to rescue declining graft function.
\end{abstract}

Keywords Chronic allograft nephropathy $\cdot$ Chronic rejection $\cdot$ Chronic allograft damage $\cdot$ Chronic allograft dysfunction $\cdot$ Protocol biopsies $\cdot$ CNI toxicity $\cdot$ CAN risk factors $\cdot$ CAN treatment $\cdot$ CADI $\cdot$ Surrogate marker

\section{Introduction}

During the 1970 s and early 1980 s, the primary focus of renal transplant research was the treatment of acute rejection (AR).

\section{S. Yilmaz $(\bowtie)$}

Division of Transplant Surgery, University of Calgary,

Foothills Medical Center Rm. 729 North Tower, 1403-29th Street NW, Calgary, AB T2N 2 T9, Canada

e-mail: serdar.yilmaz@albertahealthservices.ca
A major breakthrough in the prevention of AR occurred with the introduction of cyclosporine A (CsA), first into experimental animal studies and then into clinical practice [1] in the late 1970s. However, the dramatic improvement in short-term transplant survival in the 1980s was not reflected in the halflife of kidneys after the first post-operative year [2], and so researchers turned their attention to the reasons for long-term kidney graft loss.

In a consensus conference on chronic rejection (a term that was later replaced with chronic allograft nephropathy) during the Fourth Alexis Carrel Conference held in Kiruna, Sweden in 1992, chronic kidney allograft rejection was defined as "gradual deterioration in graft function (during the course of at least three months post-transplantation) in the absence of any other disease and necessary graft histology to confirm the diagnosis" [3]. Chronic allograft nephropathy (CAN) is therefore a clinical and pathological description and not a diagnosis, and its pathogenesis must still be clarified. This review will examine quantitative histological and functional markers as early indicators (surrogate endpoints) of CAN, risk factors associated with chronic allograft pathology, and potential treatments.

\section{Quantitative Histology and Functional Markers}

The pathologic changes of CAN are myointimal proliferation of arteries and arterioles; interstitial fibrosis with relatively mild-to-moderate mononuclear cell infiltration; significant tubular atrophy and glomerular changes, including increased mesangial matrix, glomerular basement membrane, and Bowman capsular thickening; and segmental focal glomerulosclerosis [4-6]. The histological changes of chronic allograft pathology, however, are nonspecific [7]. Similar findings may be encountered in other conditions such as kidneys from old donors, hypertension, etc. Moreover, the 
histologic features of this pathology are also found in routine biopsies of kidney transplants, even with well-functioning grafts [8].

Although it is not clear to what extent the intensity of histological changes predict a chronic deterioration of allograft function, the typical histopathologic changes of chronic allograft pathology have been well-described by several investigators. In 1992, six quantitative histologic variables (interstitial inflammation, interstitial fibrosis, glomerular mesangial matrix expansion, glomerulosclerosis, vascular intimal proliferation, and tubular atrophy) were found to be significantly associated with decline of kidney function as measured by the level of serum creatinine in both humans [8] and a rat renal allograft model [9]. The Chronic Allograft Damage Index (CADI) was constructed from the sum of the scores for those lesions. The CADI was strongly correlated to the impairment of kidney transplant function both in rodents $(\mathrm{r}=0.874)$ [10] and in human studies $(\mathrm{r}=0.717)$ [11]. This association was further supported by other independent studies $[6,12]$ and confirmed in a protocol biopsy-controlled multicenter study, where the 12-month CADI score was found to correlate with three-year graft survival [13].

From these beginnings, attempts have been made to establish consensus criteria for histological changes associated with chronic allograft pathology. In fact, the popular term "chronic rejection" was replaced by CAN to acknowledge the lack of specificity of the histologic changes described in the original Banff classification in 1991 [14]. The Banff '91 criteria for grading overall intensity of chronic allograft injury included interstitial fibrosis and tubular atrophy as assessed by visual estimates, without any numerical quantitation. These criteria also omitted the glomerular and vascular changes [14] despite the fact that glomerular and vascular changes are characteristic of CAN both in humans $[6,8,12]$ and experimental animals [9]. The Banff ' 97 classification [15] provided threshold values for chronic changes in renal allograft biopsies, which were largely adapted from the previous CADI thresholds, with minor alterations. In the Banff '05 classification, the of Banff "97 CAN grading was replaced with a new name, "IF/TA" ("interstitial fibrosis and tubular atrophy, no evidence of any specific etiology") [16]. These nonspecific terms, CAN or IF/ TA, have been used to denote the morphological changes used as surrogate markers for chronic allograft injury.

Most studies have determined that tubular atrophy and interstitial fibrosis are strongly linked and synergistic, and thus interstitial fibrosis is sufficient as a predictor without the addition of tubular atrophy. Research has also found that isolated IF/TA is only weakly correlated with graft survival, whereas IF/TA in conjunction with other histopathological parameters, such as inflammation and vascular intimal thickening, is more strongly correlated with graft outcome [17-20] suggesting that a combination of histological indicators may be superior to a limited number in predicting graft loss.
Clinical studies have revealed several clinical risk factors that are associated with chronic allograft injury [21]. Given the complex relationship between clinical risk factors and early histopathological changes, the composite and quantitative histological indices are best suited as surrogate markers for evaluating the status of the transplant [22]. As we are aware that transplant outcome is a situation where more than one pathway is critical, it is reasonable to assume that a potential surrogate marker may comprise several histological indices [23]. Therefore, the Banff ' 97 and Banff ' 05 gradings, with their current histological $[15,16]$ components for chronic allograft injury, may underestimate the severity of disease and could lead to inappropriate assessment of the degree of damage. In a study by our group [24], we assessed the prognostic values of two composite scores, CADI and the sum of the Banff Chronic score $(\mathrm{ci}+\mathrm{ct}+\mathrm{cg}+\mathrm{cv}+\mathrm{mm})$, as well as CAN grading (mild, moderate, and severe) for predicting allograft dysfunction/graft loss in 318 consecutive 6-month surveillance biopsies. The CADI score outperformed the other variables in its ability to predict graft outcome.

A drawback to the use of early histological markers to predict chronic allograft pathology is that many centers are reluctant to perform transplant protocol biopsies, given the invasive nature of the procedure. Although the risk of significant complications following renal biopsy has decreased over the years, many transplant centers appear to use early functional biomarkers as a replacement for histopathology to predict chronic allograft dysfunction. Functional biomarkers such as serum creatinine [25] and estimated glomerular filtration rate (eGFR) [26] have the clear advantage of being inexpensive and noninvasive screening tests for chronic allograft dysfunction compared with transplant protocol biopsy. Unfortunately, the widespread adoption of serum creatinine and eGFR as surrogate markers for predicting graft outcome has occurred without rigorous evaluation. In fact, Kaplan and colleagues [27] demonstrated that serum creatinine at one year was a poor predictor of allograft loss at two years (area under the receiver operator characteristic curve $[$ AUROC] $=0.63$ ). This result illustrates the scenario where a test result may have a good correlation with a clinical endpoint but fail as a surrogate marker. In a study from our institution [28], there was a relatively high prevalence of acute (interstitial inflammation, $30 \%$ ) and chronic (fibrosis, $50 \%$; tubular atrophy, $65 \%$; vascular intimal proliferation, $48 \%$; arteriolar hyalinosis, $47 \%$ ) histological changes seen in transplant protocol biopsies at 6 months from 280 patients. In contrast, renal function was relatively stable, and the average serum creatinine and eGFR levels were $122 \pm 38 \mu \mathrm{mol} / \mathrm{l}$ and $58 \pm$ $19.0 \mathrm{ml} / \mathrm{min}$, respectively, which are considered normal for transplant patients. We tested the capacity of creatinine and eGFR for predicting histological changes using area under the receiver operator characteristic curve (AUROC). The values ranged from 0.51 (representing no discrimination) to 0.62 , 
indicating that neither serum creatinine nor eGFR had predictive value for any of the individual histological parameters. Despite the obvious attractiveness of using functional markers as predictors or surrogates for chronic allograft dysfunction/ graft loss, the adoption of serum creatinine and eGFR as surrogate or predictive markers of graft outcomes has been premature.

Although substantial progress has been made toward developing histological indices that will predict or act as surrogate markers for graft outcomes, more research is needed. Development of such indices may allow both earlier intervention and provide surrogate endpoints for drug development, thus reducing the time and cost involved.

\section{Risk Factors}

There are a number of both alloantigen-dependent and alloantigen-independent risk factors that appear to contribute to chronic allograft damage/chronic allograft nephropathy. CAN is a multifactorial syndrome and represents the cumulative response to injury, regardless of the type of injury [29, 21]. Considering the heterogeneity of these risk factors, chronic allograft damage probably results from a multitude of various interacting immunological and non-immunological factors with a similar final common pathway. Taken together, it appears that CAN is not a distinct entity with a unique pathogenesis, but is rather the end result of successive episodes of AR (some of which may have been self-limiting and thus not recognized), additional injury via various mechanisms of peri- and postoperative damage, and additional non-immunologic risk factors (similar to atherosclerosis risk factors).

\section{Immunologic Risk Factors}

One or more AR episodes are the strongest predictors for long-term graft survival $[30,31]$. A single rejection episode after transplantation reduces the average half-life of first transplants from 45 to 25 years, and multiple rejection episodes decrease the half-life to five years [32].

The development of CAD in allogeneic grafts is a much more rapid process than is observed in syngeneic or isogeneic rat renal grafts, suggesting a role for an immune process in allogeneic grafts [33]. Our rat renal transplant model across strong histocompatibility barriers was entirely concordant: the more AR episodes, the less satisfactory the transplant function at 3 months and the more prominent the vascular and glomerular changes in transplant histology [34]. We also showed in the same animal model that there is a causal relationship between AR and chronic rejection and that the intensity and length of AR episodes, as well as their frequency, contribute to the development of chronic allograft damage [10].
Apart from frequency of AR, other alloantigen-dependent factors such as subclinical rejection [35•], antibody-mediated rejection [36], the presence of anti-HLA antibodies [37], vascular rejection [38], and HLA mismatches [39] show strong effects on the development of chronic allograft damage and thus long-term graft outcome.

Over the last 10 years, we have begun seeing major reductions in AR rates with the current immunosuppressive protocols. However, the reduced rates of rejection in two large series from Australia and New Zealand [38] and the U.S. [40] were not associated with significant improvement in graft survival, suggesting that factors other than $\mathrm{AR}$ are involved.

\section{Renal Calcineurin Inhibitor (CNI) Toxicity}

Calcineurin inhibitors (CNIs), such as CsA and tacrolimus, given as immunosuppressants to transplant patients have been credited for the drastic reduction in AR over the last 30 years. Since the early 1980s, CNI nephrotoxicity has been implicated as one of the major causes of chronic allograft dysfunction and late renal allograft loss, thought to be due to irreversible histological damage. More recently, however, this determination has come into question.

During the 1980s, when high doses of CsA were used, isometric vacuolization, tubular calcification, giant mitochondria, and the focal or striped form of interstitial fibrosis were considered to be diagnostic indicators of CsA nephrotoxicity [41]. More recently, hyaline arteriolar thickening has been the only morphological indicator found to distinguish patients with CNI toxicity [42, 4].Hyaline arteriolar thickening, however, is not specific to CNI toxicity. It was demonstrated in 839 non-transplant Japanese autopsy subjects that the frequency of arterial hyalinosis and arteriosclerosis increased linearly with advancing age and was associated with higher blood pressure, higher total cholesterol level, higher serum creatinine level, lower glomerular filtration rate, and proteinuria [43]. In our recently completed multicenter clinical trial with central biopsy reading, more than 400 baseline-transplant biopsies showed $34 \%$ arteriolar hyalinosis (unpublished data). It is becoming increasingly clear that arteriolar hyalinosis is linked to a multitude of underlying conditions in addition to chronic CNI use.

One difficulty in understanding the impact of CNI treatment on kidney pathology is that many studies in this area do not have appropriate comparators. In one of the earliest reports linking CNIs to native renal damage, which studied heart transplant recipients with and without CsA treatment [44, 45], the study did not include an analysis of the biopsy findings in the non-CsA group, the sample size for the renal hemodynamic studies was very small, and the patient baseline characteristics were neither defined in detail nor stratified. Subsequent studies of heart [46, 47] and liver [48, 49] 
transplant patients reported striped interstitial fibrosis, nodular arterial hyalinosis, and glomerulosclerosis in renal biopsies of non-renal transplant recipients, which were attributed to CNI toxicity. However, when we examined the patient populations in those reports, we found that the majority of patients had preexisting conditions such as hepatitis $\mathrm{C}$, chronic kidney disease, diabetes, and hypertension. Additionally, one frequently cited study suggesting CNI toxicity did not include a non-CNI-treated control group [50]; moreover, the 10-year death-censored graft survival for their patient population was excellent $(95 \%)$ with an immunosuppressant regimen that included CNI. Finally, a recent study found that the prevalence of arteriolar hyalinosis at five years was the same whether or not the patients were treated with CNI [51•]. Taken together, it is difficult to determine whether the histological lesions seen in CNI-treated transplant patients are a direct result of drug treatment or are secondary to other pathological processes.

Despite the concern that CNI nephrotoxicity may be responsible for a significant proportion of late graft dysfunction, most studies have shown that CNI-free immunosuppression provides no long-term benefit [52]. Based on scientific registry data, 6-month AR rates were significantly higher with sirolimus in combination with mycophenolate mofetil versus other regimes including CNI. Overall graft survival was significantly lower on sirolimus/mycophenolate mofetil relative to tacrolimus/mycophenolate mofetil or CsA/mycophenolate mofetil [53]. In a study of 566 patients who received kidney transplants, the frequency of chronic rejection was higher in patients who received less than $5 \mathrm{mg}$ of CsA per kilogram per day [30]. Furthermore, patients who received higher doses of CsA had better renal function for the first 3 years after transplantation [54], and patients who had lower exposure to tacrolimus had higher chronic histological damage [55], refuting the posited chronic nephrotoxic effects of CNIs.

Two recent comprehensive reviews $[56 \bullet \bullet, 57 \bullet \bullet]$ argue that CNI toxicity has been overstated. The current trend toward $\mathrm{CNI}$ avoidance, elimination, or minimization has not been based on strong evidence that CNIs can cause progressive nephropathy. While CNIs may play some role in potentiating chronic kidney injury in solid-organ transplantation, the extent of their contribution to chronic allograft injury is unknown. Moreover, the increased immunologic activity due to minimization or avoidance of CNIs may pose a greater danger to the function of renal allografts than CNI nephropathy. CNIs remain the backbone of current immunosuppression regimens and have been associated with excellent graft survival rates.

\section{Ischemia/Reperfusion Injury}

Delayed graft function and prolonged ischemia is associated with overall poor graft survival compared to grafts with immediate function in both humans [58] and animal models [59]. As a result of non-specific ischemia/reperfusion injury, the biologically uninjured transplant transforms into an inflamedinjured organ that appears to facilitate subsequent specific immunologic events; intragraft allorecognition and alloactivation by/of host T-lymphocytes result in progressive fibrosis of renal tissue [60-62].

\section{Donor and Recipient Age}

Very early studies identified donor age as a risk factor for short- and long-term graft survival. More recently, donor age was significantly associated with interstitial fibrosis, tubular atrophy, and glomerulosclerosis in implant biopsies. Additionally, among donor demographics, only donor age was found to be a significant predictor of long-term death-censored graft survival [63]. In our study, which correlated a number of clinical risk factors with histologic evidence of chronic allograft damage in one-year protocol biopsies, older donor age and younger recipient age were associated with both higher CADI score and, to varying extents, individual histopathological indices [22].

Other studies confirm that younger recipient age is a risk factor for graft survival. When all deaths were censored, recipient age $>50$ was associated with significantly better graft survival than age $<50$ [64], and better survival of renal transplants in older recipients was also demonstrated [64]. With increasing age, there was a diminishing percentage of graft loss due to immunologic causes [65]. The protective effect of higher recipient age may be related to decreased immune responsiveness and thus decreased graft loss due to immunological causes. These age-related changes in the AR rate, especially when all deaths are censored, may explain why younger recipients have shorter long-term graft survival.

\section{Hypertension}

The precise role of hypertension in chronic allograft damage has been difficult to characterize. The cause-effect relationship between hypertension and deteriorating allograft function is confounded by "reverse causation," i.e., impaired renal function leading to hypertension. Virtually all patients with renal disease are hypertensive by the time end-stage renal disease is reached. Although blood pressure may be well-controlled by dialysis, hypertension after renal transplantation is extremely common, occurring in $80 \%$ of CsA-treated patients [66]. Poorly controlled blood pressure beyond one year after transplantation is associated with poor allograft survival [66].

In experimental rat aortic allografts, hypertension was associated with a significant increase in intimal thickness involving both smooth muscle cell proliferation and collagen secretion. The angiotensin-converting-enzyme inhibitor perindopril was able to decrease the systolic blood pressure by $30 \%$ and concomitantly reduce intimal thickness by $40 \%$ [67]. It has been observed that antihypertensive drugs 
decrease the extent of glomerular mesangiolysis and glomerulosclerosis in experimental rat renal transplants [68].

\section{Hyperlipidemia}

Hypercholesterolemia has been implicated in the pathogenesis of chronic allograft damage due to histological similarities between $\mathrm{CAD}$ and atherosclerosis. In an animal experimental model of CAD, increased cholesterol intake was found to aggravate the vascular lesions [69]. In addition, clinical studies have reported an association between lipid abnormalities and several histopathological indicators of chronic renal allograft damage [70, 71]. The presence of metabolic syndrome was associated with impaired renal allograft function beyond one year post-transplant. Among its components, only systolic blood pressure and hypertriglyceridemia were independently associated with impaired renal allograft function beyond one year post-transplant [72].

\section{Glomerular Hyperfiltration}

Glomerular hyperfiltration has been identified as a risk factor [73]. Consistent with findings in non-transplant renal disease, loss of renal mass due to immunological damage and/or nonimmunologically mediated hemodynamic factors [74] lead to an increase in glomerular capillary blood flow and capillary pressure [75], and hyperfiltration in the remaining nephrons accelerates renal injury [76]. At this point, renal functional deterioration becomes a self-propagating process.

\section{Cytomegalovirus Infection}

Although there is no clear-cut evidence of the role of cytomegalovirus (CMV) infection in CAD in humans, clinical studies have shown an association between CMV infection and arteriosclerosis in heart transplants [77]. Studies in rats showed that CMV infection enhanced CAN in kidney allografts [78] and arteriosclerosis in aortic allografts [79]. In the case of rat kidney allografts, CMV infection significantly increased chronic histological lesions, which are associated with the number of AR episodes, interstitial inflammation, and the expression of intercellular adhesion molecule- 1 on vascular endothelial and tubular epithelial cells [78]. When rats receiving aortic allografts were treated with ganciclovir, the enhanced intimal response was entirely eradicated [80]. Similarly, lower rates of AR were reported at six months in a group receiving valacyclovir prophylaxis compared with placebo, primarily in donor-positive, recipient-negative sero-pairs [81].

The temporal relationship between CMV infection and AR is not clearly delineated. It is possible that CMV infection may increase the risk of AR, as suggested in the studies mentioned above; conversely, the enhanced immunosuppression during the treatment of AR may predispose the recipient to CMV infection. CMV may enhance chronic rejection by the enhancement of proinflammatory and inflammatory responses, which may or may not act in concert with acute cell-mediated rejection. This hypothesis has yet to be tested.

\section{Treatments}

Animal studies [82, 83] clearly demonstrate that, once triggered, chronic rejection becomes independent of the causative event and proceeds on its own [29]. This may also be the case for human transplantation. Since no effective therapy is currently available, CAN inevitably progresses to end-stage kidney disease. In kidney transplantation, it has been known since the early 1990s that recipients having AR episodes are at increased risk for graft loss [30]. Conversely, in the absence of an AR episode, graft survival is excellent [31]. Since prevention is clearly better than cure, optimizing the immunosuppressive regimen in organ transplantation may be the optimal prophylactic strategy today, and other pharmacological agents also show promise as adjuncts to immunotherapy. Whereas avoiding or mitigating recognized clinical risk factors are also good strategies for improving graft survival, it is clear that there are other risk factors for late graft loss that have yet to be defined.

\section{Immunosuppressive Regimens}

Potent immunosuppressive combinations to reduce inflammation, diagnosed as either subclinical [84] or clinical rejection [31], result in better graft survival, as alloimmunity remains the most common mechanism leading to graft failure [85••]. As mentioned earlier, treatment with CNIs such as cyclosporine and tacrolimus has revolutionized the area of transplantation medicine, resulting in the reduction of AR events. However, several researchers are of the view that this early survival gain is countered by perceived chronic nephrotoxicity. Regardless of whether chronic nephrotoxicity is overstated, as is the view of some authors $[56 \bullet \cdot 57 \bullet \bullet]$, it is clear that further research is needed, and drug regimens that balance the ARpreventing effect of CNIs while minimizing dose requirement are being developed [52].

Mycophenolate mofetil, which inhibits the proliferation of $\mathrm{T}$ and $\mathrm{B}$ lymphocytes, is commonly used in combination with CNIs. A systematic review of 19 studies - including a total of 3,143 patients [86] - and a single-center study [87] showed that this drug conferred a clinical benefit and reduced chronic histological changes, respectively.

Sirolimus is another immunosuppressant and vasculoprotective agent with promising albeit not yet definitive benefits. Sirolimus is a relatively weak immunosuppressive compound [29], however, and the high doses required 
may expose the patient to perioperative complications such as wound dehiscence, delayed graft function, and lymphocele. Indeed, these are factors that have prompted the discontinuation of sirolimus in many cases. Based on results of recent clinical trials in the de novo transplant setting, triple therapy with sirolimus, mycophenolate mofetil, and corticosteroids is not recommended [52], as CNIs have been associated with major improvements in early rejection outcomes in the acute phase of the transplant. However, in patients with normal/ stable kidney function after 3-6 months post-transplant, sirolimus-based regimens can be used for the agent's vasculoprotective effects to prevent further organ scarring [88]. In our institution, we perform surveillance biopsies in patients with normal/stable kidney function at around six months post-transplantation. If the biopsy shows chronic histologic changes, sirolimus is added to the therapy, replacing MMF or CNI [88].

\section{Other Pharmacological Agents}

Various pharmacological agents have shown some promise in slowing the progression of renal allograft failure in animal studies of chronic rejection. These include prostaglandin inhibitors [89], the somatostatin analog angiopeptin [90, 91], low-molecular-weight heparin in combination with CsA [92], and antihypertensive drugs $[68,93]$. The promising results, however, have not been reproduced in humans.

In a retrospective study, the use of aspirin as an adjuvant to current post-transplant treatment regimens was found to have functional benefits and improved allograft survival [94].

Retrospective studies measuring the effects of angiotensinconverting-enzyme (ACE) inhibitors or angiotensin receptor blockers (ARBs) in kidney transplant recipients reported conflicting results on allograft and patient survival. In an analysis of 17,209 renal and 1,744 heart transplant recipients, the use of ACE inhibitors/ARBs was not associated with an improvement in patient or transplant survival [95]. In contrast, another retrospective study of 2,031 patients found that the use of such agents was associated with improved long-term graft and patient survival [96]. A systematic review of randomized trials (21 trials with 1,549 patients) to determine the effect of the use of ACE-inhibitor or ARB following kidney transplantation concluded that there were insufficient data to determine the effect on patient or graft survival [97].

Dietary protein restriction has also been shown to improve the course of renal failure in chronic kidney allograft rejection [98], as it has on patients with chronic renal impairment of native kidney diseases [99].

\section{Clinical Risk Factors}

In addition to immunosuppressive protocols, avoiding clinical risk factors is a sound strategy for maximizing graft viability.
This includes avoiding sensitization, optimizing HLA matching, taking into account cold ischemia time, minimizing ischemic injury, and matching recipient and donor age. As the average age of both donors and recipients and is rising, older donors should be allocated to older recipients in order to match the recipient's shorter life expectancy. Conversely, younger recipients should receive organs from younger donors, who would have a larger nephron mass compared with older donors.

Despite our deepened understanding of clinical risk factors, it is clear that there are other determinants of graft loss that have yet to be identified. When we attempted to correlate a number of clinical risk factors with histologic evidence of CAN on protocol biopsies, we found that only $60 \%$ of the variability in chronic allograft damage could be explained by clinical risk factors [22]. Therefore, other "missing" factors must play a role in the pathogenesis of CAN.

\section{Conclusions}

The significant progress over the last two decades in renal transplantation has been driven primarily by improvements in short-term graft survival, Unfortunately, there has been little parallel improvement in long-term outcomes [100]. Although the development of better histological scores are improving efforts to detect early signs of chronic allograft pathology by histological indicators, therapies to rescue declining graft function are limited and warrant further efforts.

Acknowledgments The author would like to thank Ms. Ann Kyle for her editorial assistance in preparing this review.

\section{Compliance with Ethics Guidelines}

Conflict of Interest Serdar Yilmaz has received honoraria and grants from Astellas, Novartis, and Pfizer. He has also received payment for development of educational presentations including service on speakers' bureaus from Wyeth.

Human and Animal Rights and Informed Consent This article does not contain any studies with human or animal subjects performed by any of the authors.

\section{Reference}

Papers of particular interest, published recently, have been highlighted as:

- Of importance

•• Of major importance

1. Calne RY, White DJ, Thiru S, Evans DB, McMaster P, Dunn DC, et al. Cyclosporin A in patients receiving renal allografts from cadaver donors. Lancet. 1978;2(8104-5):1323-7. 
2. Gjertson DW. Survival trends in long-term first cadaver-donor kidney transplants. Clin Transpl. 1991:225-35.

3. Paul LC, Häyry P, Foegh M, Dennis MJ, Mihatsch MJ, Larsson E, et al. Diagnostic criteria for chronic rejection/accelerated graft atherosclerosis in heart and kidney transplants: joint proposal from the Fourth Alexis Carrel Conference on Chronic Rejection and Accelerated Arteriosclerosis in Transplanted Organs. Transplant Proc. 1993;25(2):2022-3.

4. Mihatsch MJ, Ryffel B, Gudat F. Morphological criteria of chronic rejection: differential diagnosis, including cyclosporine nephropathy. Transplant Proc. 1993;25(2):2031-7.

5. Sanfilippo F. Renal transplantation. In: Sale GE, editor. The Pathology of Organ Transplantation. Boston: Butterworths; 1990. p. 51-101.

6. Kasiske BL, Kalil RS, Lee HS, Rao KV. Histopathologic findings associated with a chronic, progressive decline in renal allograft function. Kidney Int. 1991;40(3):514-24.

7. Cornell LD, Colvin RB. Chronic allograft nephropathy. Curr Opin Nephrol Hypertens. 2005;14(3):229-34.

8. Isoniemi H, Krogerus L, von Willebrand E, Taskinen E, Ahonen J, Häyry P. Histopathological findings in well-functioning, longterm renal allografts. Kidney Int. 1992;41(1):155-60.

9. Yilmaz S, Taskinen E, Paavonen T, Mennander A, Häyry P. Chronic rejection of rat renal allograft. I. Histological differentiation between chronic rejection and cyclosporin nephrotoxicity. Transpl Int. 1992;5(2):85-95.

10. Yilmaz S, Yilmaz A, Häyry P. Chronic renal allograft rejection can be predicted by area under the serum creatinine versus time curve (AUCCr). Kidney Int. 1995;48(1):251-8.

11. Isoniemi H, Taskinen E, Häyry P. Histological chronic allograft damage index accurately predicts chronic renal allograft rejection. Transplantation. 1994;58(11):1195-8.

12. Dimeny E, Wahlberg J, Larsson E, Fellström B. Can histopathological findings in early renal allograft biopsies identify patients at risk for chronic vascular rejection? Clin Transplant. 1995;9(2):7984.

13. Yilmaz S, Tomlanovich S, Mathew T, Taskinen E, Paavonen T, Navarro M, et al. Protocol core needle biopsy and histologic Chronic Allograft Damage Index (CADI) as surrogate end point for long-term graft survival in multicenter studies. J Am Soc Nephrol. 2003;14(3):773-9.

14. Solez K, Axelsen RA, Benediktsson H, Burdick JF, Cohen AH, Colvin RB, et al. International standardization of criteria for the histologic diagnosis of renal allograft rejection: the Banff working classification of kidney transplant pathology. Kidney Int. 1993;44(2):411-22.

15. Racusen LC, Solez K, Colvin RB, Bonsib SM, Castro MC, Cavallo T, et al. The Banff 97 working classification of renal allograft pathology. Kidney Int. 1999;55(2):713-23.

16. Solez K, Colvin RB, Racusen LC, Sis B, Halloran PF, Birk PE, et al. Banff '05 Meeting Report: differential diagnosis of chronic allograft injury and elimination of chronic allograft nephropathy ('CAN'). Am J Transplant. 2007;7(3):518-26.

17. Seron D, Moreso F. Protocol biopsies in renal transplantation: prognostic value of structural monitoring. Kidney Int. 2007;72(6):690-7.

18. Cosio FG, Grande JP, Wadei H, Larson TS, Griffin MD, Stegall MD. Predicting subsequent decline in kidney allograft function from early surveillance biopsies. Am J Transplant. 2005;5(10): 2464-72.

19. Mengel M, Gwinner W, Schwarz A, Bajeski R, Franz I, Brocker V, et al. Infiltrates in protocol biopsies from renal allografts. Am J Transplant. 2007;7(2):356-65.

20. Yilmaz S, Sar A, Gonul I, Fick G, Monroy M, Salazar A et al. Interstitial inflammation and vascular intimal thickening predict long-term graft function better than Banff 97 chronic/sclerosing lesion grading (abstract \#133). American Transplant Congress; San Francisco: Am. J. Transplant.; 2007. p. 180.

21. Joosten SA, van Kooten C, Sijpkens YW, de Fijter JW, Paul LC. The pathobiology of chronic allograft nephropathy: immunemediated damage and accelerated aging. Kidney Int. 2004;65(5): 1556-9.

22. Yilmaz S, McLaughlin K, Paavonen T, Taskinen E, Monroy M, Aavik E, et al. Clinical predictors of renal allograft histopathology: a comparative study of single-lesion histology versus a composite, quantitative scoring system. Transplantation. 2007;83(6):671-6.

23. Lachenbruch PA, Rosenberg AS, Bonvini E, Cavaille-Coll MW, Colvin RB. Biomarkers and surrogate endpoints in renal transplantation: present status and considerations for clinical trial design. Am J Transplant. 2004;4(4):451-7.

24. Yilmaz S, Sar A, Gonul I, Monroy M, Salazar A, Tibbles L et al., editors. Comparison of composite histologic scores with Banff 97 chronic/sclerosing lesion grading for prediction of progressive allograft dysfunction/graft loss (abstract \#134). American Transplant Congress; 2007; San Francisco: Am. J. Transplant.

25. Hariharan S, McBride MA, Cherikh WS, Tolleris CB, Bresnahan BA, Johnson CP. Post-transplant renal function in the first year predicts long-term kidney transplant survival. Kidney Int. 2002;62(1):311-8.

26. Salvadori M, Rosati A, Bock A, Chapman J, Dussol B, Fritsche L, et al. Estimated one-year glomerular filtration rate is the best predictor of long-term graft function following renal transplant. Transplantation. 2006;81(2):202-6.

27. Kaplan B, Schold J, Meier-Kriesche HU. Poor predictive value of serum creatinine for renal allograft loss. Am J Transplant. 2003;3(12):1560-5.

28. Yilmaz S, Isik I, Afrouzian M, Monroy M, Sar A, Benediktsson H, et al. Evaluating the accuracy of functional biomarkers for detecting histological changes in chronic allograft nephropathy. Transpl Int. 2007;20(7):608-15.

29. Häyry P, Yilmaz S, Vamvakopoulos J, Aavik E. Pathology and pathophysiology of chronic rejection. Curr Opin Organ Transplant. 2006;11(3):296-303.

30. Almond PS, Matas A, Gillingham K, Dunn DL, Payne WD, Gores $\mathrm{P}$, et al. Risk factors for chronic rejection in renal allograft recipients. Transplantation. 1993;55(4):752-6. discussion 6-7.

31. Humar A, Hassoun A, Kandaswamy R, Payne WD, Sutherland DE, Matas AJ. Immunologic factors: the major risk for decreased long-term renal allograft survival. Transplantation. 1999;68(12): $1842-6$.

32. Matas AJ, Gillingham KJ, Payne WD, Najarian JS. The impact of an acute rejection episode on long-term renal allograft survival (t1/ 2). Transplantation. 1994;57(6):857-9.

33. Tullius SG, Heemann UW, Hancock WW, Azuma H, Tilney NL. Long-term kidney isografts develope functional and morphological changes that mimic those of chronic allograft rejection. Ann Surg. 1994;220(4):425-43.

34. Yilmaz S, Häyry P. The impact of acute episodes of rejection on the generation of chronic rejection in rat renal allografts. Transplantation. 1993;56(5):1153-6.

35. Thierry A, Thervet E, Vuiblet V, Goujon JM, Machet MC, Noel LH, et al. Long-term impact of subclinical inflammation diagnosed by protocol biopsy one year after renal transplantation. Am J Transplant. 2011;11(10):2153-61. The result of this protocol biopsy study found that subclinical inflamation at 1-year postransplantation is associated with worsening renal function is more frequent in sirolimus treated versus CNI treated patients.

36. Sellares J, de Freitas DG, Mengel M, Reeve J, Einecke G, Sis B, et al. Understanding the causes of kidney transplant failure: the dominant role of antibody-mediated rejection and nonadherence. Am J Transplant. 2012;12(2):388-99. 
37. Mao Q, Terasaki PI, Cai J, Briley K, Catrou P, Haisch C, et al. Extremely high association between appearance of HLA antibodies and failure of kidney grafts in a five-year longitudinal study. Am J Transplant. 2007;7(4):864-71.

38. McDonald S, Russ G, Campbell S, Chadban S. Kidney transplant rejection in Australia and New Zealand: relationships between rejection and graft outcome. Am J Transplant. 2007;7(5):1201-8.

39. Gilks WR, Gore SM, Bradley BA. Renal transplant rejection. Transient immunodominance of HLA mismatches. Transplantation. 1990;50(1):141-6.

40. Meier-Kriesche HU, Schold JD, Srinivas TR, Kaplan B. Lack of improvement in renal allograft survival despite a marked decrease in acute rejection rates over the most recent era. Am J Transplant. 2004;4(3):378-83.

41. Mihatsch MJ, Thiel G, Ryffel B. Histopathology of cyclosporine nephrotoxicity. Transplant Proc. 1988;20(3 Suppl 3):759-71.

42. Solez K, Racusen LC, Marcussen N, Slatnik I, Keown P, Burdick $\mathrm{JF}$, et al. Morphology of ischemic acute renal failure, normal function, and cyclosporine toxicity in cyclosporine-treated renal allograft recipients. Kidney Int. 1993;43(5):1058-67.

43. Kubo M, Kiyohara Y, Kato I, Tanizaki Y, Katafuchi R, Hirakata H, et al. Risk factors for renal glomerular and vascular changes in an autopsy-based population survey: the Hisayama study. Kidney Int. 2003;63(4):1508-15.

44. Myers BD, Ross J, Newton L, Luetscher J, Perlroth M. Cyclosporine-associated chronic nephropathy. N Engl J Med. 1984;311(11):699-705.

45. Myers BD, Newton L, Boshkos C, Macoviak JA, Frist WH, Derby GC, et al. Chronic injury of human renal microvessels with low-dose cyclosporine therapy. Transplantation. 1988;46(5):694703 .

46. Bertani T, Ferrazzi P, Schieppati A, Ruggenenti P, Gamba A, Parenzan L, et al. Nature and extent of glomerular injury induced by cyclosporine in heart transplant patients. Kidney Int. 1991;40(2): 243-50.

47. Myers BD, Newton L. Cyclosporine-induced chronic nephropathy: an obliterative microvascular renal injury. J Am Soc Nephrol. 1991;2(2 Suppl 1):S45-52.

48. Pillebout E, Nochy D, Hill G, Conti F, Antoine C, Calmus Y, et al. Renal histopathological lesions after orthotopic liver transplantation (OLT). Am J Transplant. 2005;5(5):1120-9.

49. Kim JY, Akalin E, Dikman S, Gagliardi R, Schiano T, Bromberg J, et al. The variable pathology of kidney disease after liver transplantation. Transplantation. 2010;89(2):215-21.

50. Nankivell BJ, Borrows RJ, Fung CL, O'Connell PJ, Allen RD, Chapman JR. The natural history of chronic allograft nephropathy. N Engl J Med. 2003;349(24):2326-33.

51. Stegall MD, Park WD, Larson TS, Gloor JM, Cornell LD, Sethi S, et al. The histology of solitary renal allografts at 1 and 5 years after transplantation. Am J Transplant. 2011;11(4):698-707. The result of this protocol biopsy study found that the prevalence of moderate or severe arteriolar hyalinosis was similar in tacrolimus and calcineurin inhibitor-free immunosuppression.

52. Ekberg H, Tedesco-Silva H, Demirbas A, Vítko S, Nashan B, Gürkan A, et al. Reduced exposure to calcineurin inhibitors in renal transplantation. N Engl J Med. 2007;357(25):2562-75.

53. Srinivas TR, Schold JD, Guerra G, Eagan A, Bucci CM, MeierKriesche HU. Mycophenolate mofetil/sirolimus compared to other common immunosuppressive regimens in kidney transplantation. Am J Transplant. 2007;7(3):586-94.

54. Burke Jr JF, Pirsch JD, Ramos EL, Salomon DR, Stablein DM, Van Buren DH, et al. Long-term efficacy and safety of cyclosporine in renal-transplant recipients. N Engl J Med. 1994;331(6):35863.

55. Naesens M, Lerut E, Damme BV, Vanrenterghem Y, Kuypers DR. Tacrolimus exposure and evolution of renal allograft histology in the first year after transplantation. Am J Transplant. 2007;7(9): 2114-23.

56.• Matas AJ. Chronic progressive calcineurin nephrotoxicity: an overstated concept. Am J Transplant. 2011;11(4):687-92. This review article provides in depth analysis of literature about CNI chronic toxicity.

57.• Issa N, Kukla A, Ibrahim HN. Calcineurin inhibitor nephrotoxicity: a review and perspective of the evidence. Am J Nephrol. 2013;37(6):602-12. This review article provides in depth analysis of literature about CNI chronic toxicity.

58. Najarian JS, Gillingham KJ, Sutherland DE, Reinsmoen NL, Payne WD, Matas AJ. The impact of the quality of initial graft function on cadaver kidney transplants. Transplantation. 1994;57(6):812-6.

59. Yilmaz S, Paavonen T, Häyry P. Chronic rejection of rat renal allografts. II. The impact of prolonged ischemia time on transplant histology. Transplantation. 1992;53(4):823-7.

60. Land W, Messmer K. The impact of ischemia/reperfusion injury on specific and non-specific, early and late chronic events after organ transplantation. Transpl Rev. 1996;10:108-27.

61. Gueler F, Gwinner W, Schwarz A, Haller H. Long-term effects of acute ischemia and reperfusion injury. Kidney Int. 2004;66(2): 523-7.

62. Bidmon B, Kratochwill K, Rusai K, Kuster L, Herzog R, Eickelberg $\mathrm{O}$, et al. Increased immunogenicity is an integral part of the heat shock response following renal ischemia. Cell Stress Chaperones. 2012;17(3):385-97.

63. De Vusser K, Lerut E, Kuypers D, Vanrenterghem Y, Jochmans I, Monbaliu D, et al. The predictive value of kidney allograft baseline biopsies for long-term graft survival. J Am Soc Nephrol. 2013;24(11):1913-23.

64. Matas AJ, Gillingham KJ, Sutherland DE. Half-life and risk factors for kidney transplant outcome-importance of death with function. Transplantation. 1993;55(4):757-61.

65. Yuge J, Cecka JM. Sex and age effects in renal transplantation. Clin Transpl. 1991:257-67.

66. Ponticelli C, Montagnino G, Aroldi A, Angelini C, Braga M, Tarantino A. Hypertension after renal transplantation. Am J Kidney Dis. 1993;21(5 Suppl 2):73-8.

67. Michel JB, Plissonnier D, Bruneval P. Effect of perindopril on the immune arterial wall remodeling in the rat model of arterial graft rejection. Am J Med. 1992;92(4B):39S-46.

68. Benediktsson H, Chea R, Davidoff A, Paul LC. Antihypertensive drug treatment in chronic renal allograft rejection in the rat. Effect on structure and function. Transplantation. 1996;62(11): 1634-42.

69. Räisanen-Sokolowski A, Tilly-Kiesi M, Ustinov J, Mennander A, Paavonen T, Tikkanen MJ, et al. Hyperlipidemia accelerates allograft arteriosclerosis (chronic rejection) in the rat. Arterioscler Thromb. 1994;14(12):2032-42.

70. Dimeny E, Tufveson G, Lithell H, Larsson E, Siegbahn A, Fellström B. The influence of pretransplant lipoprotein abnormalities on the early results of renal transplantation. Eur J Clin Invest. 1993;23(9):572-9.

71. Isoniemi H, Nurminen M, Tikkanen MJ, von Willebrand E, Krogerus L, Ahonen J, et al. Risk factors predicting chronic rejection of renal allografts. Transplantation. 1994;57(1):68-72.

72. de Vries AP, Bakker SJ, van Son WJ, van der Heide JJ, Ploeg RJ, The HT, et al. Metabolic syndrome is associated with impaired long-term renal allograft function; not all component criteria contribute equally. Am J Transplant. 2004;4(10):1675-83.

73. Brenner BM, Cohen RA, Milford EL. In renal transplantation, one size may not fit all. J Am Soc Nephrol. 1992;3(2):162-9.

74. Brenner BM, Milford EL. Nephron underdosing: a programmed cause of chronic renal allograft failure. Am J Kidney Dis. 1993;21(5 Suppl 2):66-72. 
75. Hostetter TH, Olson JL, Rennke HG, Venkatachalam MA, Brenner BM. Hyperfiltration in remnant nephrons: a potentially adverse response to renal ablation. Am J Physiol. 1981;241(1):F85-93.

76. Terasaki PI, Koyama H, Cecka JM, Gjertson DW. The hyperfiltration hypothesis in human renal transplantation. Transplantation. 1994;57(10):1450-4.

77. Grattan MT, Moreno-Cabral CE, Starnes VA, Oyer PE, Stinson $\mathrm{EB}$, Shumway NE. Cytomegalovirus infection is associated with cardiac allograft rejection and atherosclerosis. JAMA. 1989;261(24):3561-6.

78. Yilmaz S, Koskinen PK, Kallio E, Bruggeman CA, Hayry PJ, Lemstrom KB. Cytomegalovirus infection-enhanced chronic kidney allograft rejection is linked with intercellular adhesion molecule-1 expression. Kidney Int. 1996;50(2):526-37.

79. Lemström KB, Aho PT, Bruggeman CA, Hayry PJ. Cytomegalovirus infection enhances mRNA expression of platelet-derived growth factor-BB and transforming growth factor-beta 1 in rat aortic allografts. Possible mechanism for cytomegalovirus-enhanced graft arteriosclerosis. Arterioscler Thromb. 1994;14(12):2043-52.

80. Lemström KB, Bruning JH, Bruggeman CA, Koskinen PK, Aho PT, Yilmaz S, et al. Cytomegalovirus infection-enhanced allograft arteriosclerosis is prevented by DHPG prophylaxis in the rat. Circulation. 1994;90(4):1969-78.

81. Lowance D, Neumayer HH, Legendre CM, Squifflet JP, Kovarik $\mathrm{J}$, Brennan PJ, et al. Valacyclovir for the prevention of cytomegalovirus disease after renal transplantation. International Valacyclovir Cytomegalovirus Prophylaxis Transplantation Study Group. N Engl J Med. 1999;340(19):1462-70.

82. Mennander A, Häyry P. Reversibility of allograft arteriosclerosis after retransplantation to donor strain. Transplantation. 1996;62(4):526-9.

83. Schmid C, Heemann U, Tilney NL. Retransplantation reverses mononuclear infiltration but not myointimal proliferation in a rat model of chronic cardiac allograft rejection. Transplantation. 1996;61(12):1695-9.

84. Rush D, Nickerson P, Gough J, McKenna R, Grimm P, Cheang M, et al. Beneficial effects of treatment of early subclinical rejection: a randomized study. J Am Soc Nephrol. 1998;9(11):2129-34.

85.• El-Zoghby ZM, Stegall MD, Lager DJ, Kremers WK, Amer H, Gloor JM, et al. Identifying specific causes of kidney allograft loss. Am J Transplant. 2009;9(3):527-35. This study provides a through evidence of how alloimmunity was the most common mechansim leading to failure of 330 graft out of 1317 conventonal kidney transplant.

86. Knight SR, Russell NK, Barcena L, Morris PJ. Mycophenolate mofetil decreases acute rejection and may improve graft survival in renal transplant recipients when compared with azathioprine: a systematic review. Transplantation. 2009;87(6):785-94.

87. Nankivell BJ, Wavamunno MD, Borrows RJ, Vitalone M, Fung $\mathrm{CL}$, Allen RD, et al. Mycophenolate mofetil is associated with altered expression of chronic renal transplant histology. Am J Transplant. 2007;7(2):366-76.
88. Yilmaz S, Sar A. Pathogenesis and management of chronic allograft nephropathy. Drugs. 2008;68 Suppl 1:21-31.

89. Teraoka S, Takahashi K, Toma H, Ota K. Controlled prospective study of treatment for chronic rejection after kidney transplantation by thromboxane synthetase inhibitor. Transplant Proc. 1993;25(2): 2085-6.

90. Foegh ML, Khirabadi BS, Chambers E, Amamoo S, Ramwell PW. Inhibition of coronary artery transplant atherosclerosis in rabbits with angiopeptin, an octapeptide. Atherosclerosis. 1989;78(2-3):229-36.

91. Häyry P, Raisanen A, Ustinov J, Mennander A, Paavonen T. Somatostatin analog lanreotide inhibits myocyte replication and several growth factors in allograft arteriosclerosis. FASEB J. 1993;7(11):1055-60.

92. Plissonnier D, Amichot G, Lecagneux J, Duriez M, Gentric D, Michel JB. Additive and synergistic effects of a low-molecularweight, heparin-like molecule and low doses of cyclosporin in preventing arterial graft rejection in rats. Arterioscler Thromb. 1993;13(1):112-9.

93. Szabo A, Lutz J, Schleimer K, Antus B, Hamar P, Philipp T, et al. Effect of angiotensin-converting enzyme inhibition on growth factor mRNA in chronic renal allograft rejection in the rat. Kidney Int. 2000;57(3):982-91.

94. Grotz W, Siebig S, Olschewski M, Strey CW, Peter K. Low-dose aspirin therapy is associated with improved allograft function and prolonged allograft survival after kidney transplantation. Transplantation. 2004;77(12):1848-53.

95. Opelz G, Zeier M, Laux G, Morath C, Dohler B. No improvement of patient or graft survival in transplant recipients treated with angiotensin-converting enzyme inhibitors or angiotensin II type 1 receptor blockers: a collaborative transplant study report. J Am Soc Nephrol. 2006;17(11):3257-62.

96. Heinze G, Mitterbauer C, Regele H, Kramar R, Winkelmayer WC, Curhan GC, et al. Angiotensin-converting enzyme inhibitor or angiotensin II type 1 receptor antagonist therapy is associated with prolonged patient and graft survival after renal transplantation. J Am Soc Nephrol. 2006;17(3):889-99.

97. Hiremath S, Fergusson D, Doucette S, Mulay AV, Knoll GA. Renin angiotensin system blockade in kidney transplantation: a systematic review of the evidence. Am J Transplant. 2007;7(10): 2350-60.

98. Salahudeen AK, Hostetter TH, Raatz SK, Rosenberg ME. Effects of dietary protein in patients with chronic renal transplant rejection. Kidney Int. 1992;41(1):183-90.

99. Brenner BM, Meyer TW, Hostetter TH. Dietary protein intake and the progressive nature of kidney disease: the role of hemodynamically mediated glomerular injury in the pathogenesis of progressive glomerular sclerosis in aging, renal ablation, and intrinsic renal disease. N Engl J Med. 1982;307(11): 652-9.

100. Lamb KE, Lodhi S, Meier-Kriesche HU. Long-term renal allograft survival in the United States: a critical reappraisal. Am J Transplant. 2011;11(3):450-62. 